DRUG METABOLISM

\section{Small but significant}

The crystal structure of arguably the most important enzyme in drug metabolism -

cytochrome P450 3A4 (CYP3A4) - has

been published, and with it comes the

surprise discovery that its active site is much smaller than previously thought. Although the overall structure of the enzyme is characteristic of the P450 family, this paper describes several unique features that provide new insights into the ability of CYP3A4 to metabolize a wide variety of diverse substrates.

CYP3A4 metabolizes $50 \%$ of all marketed drugs - more than any other P450 isoform - and the diversity of its substrates indicate that it is capable of metabolizing several compounds simultaneously.
In the absence of a crystal structure, understanding the complexity of CYP3A4 substrate binding and conformational change has relied on the use of homology models. However, these have limited use because the sequence similarity between P450s is low — just 24\% between CYP3A4 and the recently published CYP2C9.

Writing in Science, Harren Jhoti and colleagues describe three structures of CYP3A4: unliganded, bound to a substrate (progesterone) and bound to an inhibitor (metyrapone). They report that there are some unique features of CYP3A4 that could help in the design of drugs with improved metabolic and toxicity profiles.

One particular feature unique to CYP3A4 is the observed 'Phe-cluster' of seven phenylalanine residues that form a hydrophobic core pointing towards the active site. Some of the residues that form this cluster have been shown by mutational analysis to have a role in CYP3A4 activity. The presence of this cluster means that the accessible volume of the active site is much smaller than would be expected considering the large molecular size of some CYP3A4 substrates. The authors propose that conformational change of the Phe-cluster could reposition phenylalanine residues, resulting in an extended active site that is capable of binding more than one substrate. In addition, the active site in CYP3A4 has greater access to the haem moiety compared with CYP2C9. This proximity could enable two substrate molecules to have access to reactive oxygen, potentially providing the means for CYP3A4 to metabolize more than one substrate simultaneously.

The authors investigated whether conformational change was a prerequisite for substrate or inhibitor binding. They were surprised to find that there was no conformational change in metyrapone-bound CYP3A4, but postulated that this might be because metyrapone is a small molecule that the CYP3A4 active site could accommodate easily. To test this hypothesis, they studied the

\title{
Outside the site: a lateral approach to phosphatase inhibition
}

Allosteric inhibition of the protein tyrosine phosphatase-1B (PTP1B) prevents formation of the active conformation of the enzyme by blocking mobility of the catalytic loop, according to research published in the August issue of Nature Structural and Molecular Biology. PTP1B is an attractive target for the therapeutic intervention of metabolic syndromes such as type 2 diabetes and obesity - states that show hallmark resistance to the hormones insulin and leptin - because it is a negative regulator of the relevant hormone receptors. The catalytic site of PTP1B has so far proven intractable to small-molecule drugs, making allosteric inhibition an appealing strategy.

PTP1B is well validated as a negative regulator of insulin receptor phosphorylation and signalling. Mice deficient for the protein are resistant to obesity, do not develop type 2 diabetes and show no phenotypic abnormalities. In addition, antidiabetic and anti-obesity effects have been demonstrated by blocking expression of PTP1B with antisense oligonucleotides and small-molecule inhibitors. In spite of this, the nature of the highly charged active site and adjacent shallow phosphotyrosine-binding pocket in $\mathrm{PTP} 1 \mathrm{~B}$ remain challenging aspects to overcome, and no PTP1B-specific compounds have yet entered clinical trials. Hansen and colleagues screened for nontyrosine phosphate-like inhibitors, and identified a number of compounds that were not competitive with the PTP1B substrate. High-resolution structures of PTP1B complexed with a number of inhibitors revealed that instead of blocking the catalytic site, the small molecules were binding to a novel allosteric site on the enzyme. Binding at this site maintains the enzyme in an inactive form, because the compounds block interactions that are necessary for movement of the catalytic loop. Other phosphatases use a similar catalytic mechanism and might therefore also be candidates for allosteric inhibition.

Most competitive PTP mimetics have low cell permeability, so the authors tested whether the allosteric inhibitors could cross the cell membrane and activate insulin signalling. Overexpression of PTP1B in whole-cell lysates of Chinese hamster ovary cells expressing the human insulin receptor led to reduced insulin-induced receptor phosphorylation; however, phosphorylation was restored in the presence of the allosteric inhibitors. Demonstration of allosteric inhibition of PTP1B indicates that this strategy could be a good approach for inhibiting other phosphatases. Moreover, the residues lining the allosteric site are not well conserved among phosphatases, which means that this strategy could be a good approach for developing inhibitors with the requisite selectivity and cell permeability.

Melanie Brazil

(D) References and links ORIGINAL RESEARCH PAPER Wiesmann, C. et al. Allosteric inhibition of protein tyrosine phosphatase 1B. Nature Struct. Mol. Biol. 11, 730-737 (2004) FURTHER READING

Johnson, T. O., Ermolieff, J. \& Jirousek, M. R. Protein tyrosine phosphatase $1 \mathrm{~B}$ inhibitors for diabetes. Nature Rev. Drug Discov. 1, 696-709 (2002) | Christopoulos, A. Allosteric binding sites on cell-surface receptors: novel targets for drug discovery. Nature Rev. Drug Discov. 1, 198-210 (2002) 
